\title{
Galaxy Genesis - Unravelling the Epoch of Dissipation in the Early Disk
}

\author{
Joss Bland-Hawthorn ${ }^{1,3}$ and Ken C. Freeman ${ }^{2}$ \\ ${ }^{1}$ Anglo-Australian Observatory, Eastwood NSW 2122, Australia \\ ${ }^{2}$ Mount Stromlo Observatory, Australia National University, Weston Creek ACT 2611, Australia \\ ${ }^{3}$ E-mail: jbh@aao.gov.au
}

Received 2004 February 20, accepted 2004 March 22

\begin{abstract}
How did the Galactic disk form and can the sequence of events ever be unravelled from the vast stellar inventory? This will require that some of the residual inhomogeneities from prehistory escaped the dissipative process at an early stage. Fossil hunting to date has concentrated mostly on the stellar halo, but a key source of information will be the thick disk. This is believed to be a 'snap frozen' relic which formed during or shortly after the last major epoch of dissipation, or it may have formed from infalling systems early in the life of the Galaxy. As part of the KAOS Galaxy Genesis project, we explore the early history of the halo and the thick disk by looking for discrete substructures, either due to infall or in situ star formation, through chemical tagging. This will require high signal-to-noise echelle spectroscopy of up to a million stars throughout the disk. Our program has a short-term and a long-term goal.

The short-term goal is to quantify the size and structure of the multi-dimensional chemical abundance space $(\mathcal{C}$-space) for all major components of the Galaxy. We seek to establish how many axes in $\mathcal{C}$-space are decoupled and have large intrinsic dispersions. A critical test of chemical tagging in the short term is that stellar streams in the halo, identified from detailed phase space information, are highly localised in $\mathcal{C}$-space, or are confined to chemical tracks. These trajectories presuppose that stars form in a closed box through progressive enrichments of the gas, leading to stars dispersed along a narrow track in a complex chemical space. The long-term goal is to identify unique chemical signatures in the thick disk, originating from different formation sites, for star clusters which have long since dispersed. This will require precise chemical abundances for heavy elements such that a star can be localised to a discrete point in $\mathcal{C}$-space. If the star clusters originally formed outside the Galaxy in a bound infalling system, the stellar abundances may fall along a chemical track, rather than a discrete point in $\mathcal{C}$-space.
\end{abstract}

Keyword: Galaxy: kinematics and dynamics

\section{Introduction}

At the heart of a successful theory of galaxy formation must lie a detailed physical understanding of the dissipational processes which form spiral galaxies. The disk is the defining stellar component of disk galaxies, and understanding its formation is in our view the most important goal of galaxy-formation theory. Although much of the information about the pre-disk state of the baryons has been lost in the dissipative process, some tracers are likely to remain.

What do we mean by the reconstruction of early galactic history? We seek a detailed physical understanding of the sequence of events which led to the Milky Way. Ideally, we would want to associate components of the Galaxy to elements of the protocloud - the baryon reservoir which fuelled the stars in the Galaxy.

For many halo stars, and some outer bulge stars, this may be possible with phase space information. But for much of the bulge and the disk, secular processes cause the populations to become relaxed (i.e. the integrals of motion are partially randomised). In order to have any chance of unravelling disk formation, we must explore chemical signatures in the stellar spectrum.

The exponential thin disk, with a vertical scale height of about $300 \mathrm{pc}$, is the most conspicuous component in edge-on disk galaxies. The thin disk is believed to be the end product of the quiescent dissipation of most of the baryons and contains almost all of the baryonic angular momentum. Many disk galaxies show a second fainter disk component with a longer scale height (typically about $1 \mathrm{kpc}$ ); this is known as the thick disk (Schwarzkopf \& Dettmar 2000; Dalcanton \& Bernstein 2002). The Milky Way has a significant thick disk (Gilmore \& Reid 1983): Its surface brightness is about $25 \%$ of the thin disk's surface brightness and its stars are significantly more metal-poor than the stars of the thin disk.

The galactic thick disk is currently believed to arise from heating of the early stellar disk by one or more accretion events although other possible origins have been discussed (Gilmore, Wyse, \& Kuijken 1989; Gilmore, Wyse, \& Jones 1995). It is composed of only old stars, with ages greater than $10 \mathrm{Gyr}$, equivalent to forming at 
a redshift of $z \geq 1$ (Wyse 2000). Furthermore, the thick disk is chemically distinct from the thin disk at low metallicity (Fuhrmann 1998; Feltzing, Bensby, \& Lundstrom $2003)$, in the sense that $[\alpha / \mathrm{Fe}]$ is enhanced relative to the thin disk, although solar values are seen at higher metallicity. The thick disk may be one of the most significant components for studying signatures of galaxy formation because it presents a 'snap frozen' relic of the state of the (heated) early disk.

In the discussion which follows, we make reference to the KAOS project (see http://www.noao.edu/kaos) which proposes a highly multiplexed wide-field multi-object spectrograph for the Gemini Observatory. Full specifications for the proposed instrument can be found at the above web site.

\section{Unravelling a Dissipative Process}

In order to follow the sequence of events involved in dissipation, we propose that the critical components which need to be re-assembled are the individual star clusters which formed at each stage. Since most stars are born in dense clusters, the formation and evolution of galaxies today must involve millions of discrete cluster events throughout their history. We would like to establish the evolving mass function of star clusters, their chemical composition, formation, and survival rate as a function of cosmic time. Galaxy-wide enrichment from the fallout of nuclear winds or mergers would be evident in the fossil record of reconstructed star clusters, assuming these provide an unbiased sampling of cosmic time regardless of the star-formation history.

But how are we to reconstruct star clusters which have long since dispersed? It will be necessary to tag individual stars to their parent cloud through unique chemical signatures shared by these stars, assuming these exist.

We now discuss our basic strategy for "chemical tagging' (Freeman \& Bland-Hawthorn 2002, hereafter FBH). High-resolution spectroscopy at high signal-to-noise ratio of many stellar types reveals an extraordinarily complex pattern of spectral lines. The spectral lines carry key information on element abundances that make up the stellar atmosphere. Many of these elements cannot arise through normal stellar evolution, and therefore must reflect conditions in the progenitor cloud at the time of its formation.

\subsection{Primary Requirements of Chemical Tagging}

Our long-term goal is to chemically tag stars into coeval groups, i.e. to identify individual members of star clusters which have long since dispersed. For unique chemical signatures to exist, there are several key requirements $(\mathrm{FBH})$ :

1. Most stars must be born in dense star clusters.

2. Most dense star clusters must be chemically uniform in key elements.

3. Key chemical elements must reflect the cloud composition of the progenitor cloud.
4. Key chemical elements must not be rigidly coupled (i.e. vary in lock step), and there must be sufficient abundance dispersion in key elements to allow for unique groups (reflecting unique sites of formation) to be readily identified.

5. There must be a contiguous spectral window which contains the necessary information on key elements for chemical tagging.

We discuss each of these requirements, or conditions, in turn. Conditions 1, 2, 3, and 5 appear to be supported by observation. Condition 4 is the most uncertain largely because stellar abundance surveys to date target either too few stars or too few chemical elements.

Condition 1. Most stars are born within rich clusters of many hundreds to many thousands of stars (Clarke, Bonnell, \& Hillenbrand 2000; Carpenter 2000). This essential fact is supported by many studies from optical, infrared, millimeter, and radio surveys.

Condition 2. The widely held view that open clusters are chemically uniform can be traced to classic work on Hyades by Conti et al. (1965). But, until recently, very little was known about detailed heavy element abundance work on open clusters with rigorous membership established by reliable astrometry or accurate radial velocities $\left(<0.5 \mathrm{~km} \mathrm{~s}^{-1}\right)$, so as to minimise 'pollution' from stars not associated with the cluster (e.g. Quillen 2002).

There have been several recent studies of open clusters which have established that open clusters are indeed highly uniform. In a detailed analysis of $55 \mathrm{~F}-\mathrm{K}$ dwarfs in Hyades, Paulson, Sneden, \& Cochran (2003) find that the abundance variations in $\mathrm{Si}, \mathrm{Ti}, \mathrm{Na}, \mathrm{Mg}, \mathrm{Ca}$, and $\mathrm{Zn}$ with respect to $\mathrm{Fe}$, and in $[\mathrm{Fe} / \mathrm{H}]$, are within the measurement errors $(<0.04 \mathrm{dex})$.

There is remarkably little theoretical work which addresses the chemical uniformity of star clusters. In theoretical work on star formation, chemical uniformity is almost always a prior assumption. Are we to expect chemical uniformity among low-mass stars in an open cluster? To date, this question has not been adequately answered.

McKee \& Tan (2002) propose that high-mass stars form in the cores of strongly self-gravitating and turbulent gas clouds. Two possible routes to chemical uniformity is that all stars form at the same instance from a chemically uniform cloud, or that the low-mass stars form outside of the core shortly after the supernovae have uniformly enriched the cloud. The precise sequence of events which give rise to open clusters is a topic of great interest and heated debate in contemporary astrophysics (e.g. Stahler, Palla, \& Ho 2000).

Condition 3. It is generally believed that r-process elements (e.g. Sm, Eu, Gd, Tb, Dy, Ho) cannot be formed during quiescent stellar evolution. While some doubts remain, the most likely sites for the r-process appears to be Type II supernovae (SNe II), as originally suggested by Burbidge et al. (1957) (see also Wallerstein et al. 1997). Therefore, r-process elements measured from stellar atmospheres reflect conditions in the progenitor cloud. 


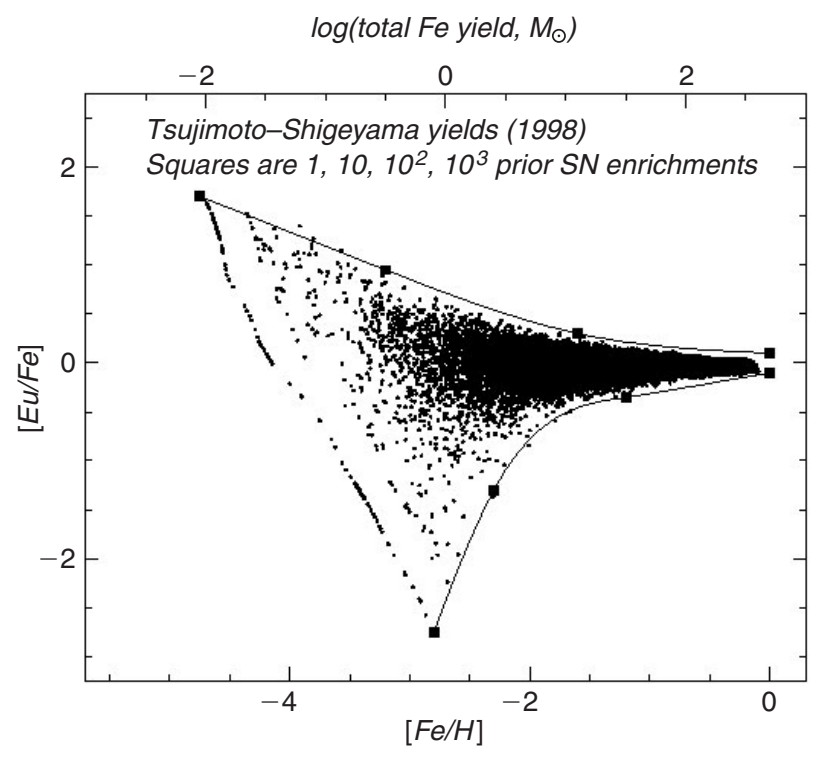

Figure 1 An illustration of how successive chemical enrichments by supernovae can lead to low-mass stars with a wide scatter in chemical abundances which converge to a universal value. The model assumes a constant star-formation rate in a closed box. The stars are formed according to a Salpeter mass function and the yields are taken from models by Tsujimoto \& Shigeyama (1998). Only $1 \%$ of the stars produced in the simulation are shown after the initial burst; we show all the stars at $t=0$.

The same is believed to be true for most of the $\alpha$ elements since these are produced in the hydrostatic burning phase of the pre-supernova star.

In contrast, the s-process elements (e.g. $\mathrm{Sr}, \mathrm{Zr}, \mathrm{Ba}, \mathrm{Ce}$, $\mathrm{La}, \mathrm{Pb})$ are thought to arise from the He-burning phase of intermediate to low-mass $(\mathrm{AGB})$ stars $\left(M<10 \mathrm{M}_{\odot}\right)$, although at the lowest metallicities, trace amounts are likely to arise from high-mass stars (Burris et al. 2000; Rauscher et al. 2002).

Condition 4. During the past four decades, evidence has gradually accumulated for a large dispersion in metal abundances $[\mathrm{X} / \mathrm{Fe}]$ (particularly n-capture elements) in low-metallicity stars relative to solar abundances (Wallerstein et al. 1963; Pagel 1965; Spite \& Spite 1978; Truran 1981; Luck \& Bond 1985; Clayton 1988; Gilroy et al. 1988; McWilliam et al. 1995; Norris, Ryan, \& Beers 1996; Burris et al. 2000). Elements like Sr, Ba, and $\mathrm{Eu}$ show a 300-fold dispersion (see Fig. 21 of Wallerstein et al. 1997), although $[\alpha / \mathrm{Fe}]$ dispersions are typically an order of magnitude smaller.

These observations have been used to constrain detailed supernova models, which in turn show how different yields arise as a function of progenitor mass, progenitor metallicity, mass cut (what gets ejected compared to what falls back towards the compact central object), and detonation details. These models help to explain the smaller dispersions in $\alpha$ elements: The $\alpha$ yields are not dependent on the mass cut or details of the fallback/explosion mechanism, which leads to a smaller dispersion at low metallicity.

Is there evidence that some elements are decoupled? The element abundances $[\mathrm{X} / \mathrm{Fe}]$ show three main peaks at $Z \sim 26, Z \sim 52$, and $Z \sim 78$ (Sneden et al. 2001a,b). There have been suggestions that the r-process gives rise to random abundance patterns (e.g. Goriely \& Arnould 1996), but this is not supported by new observations of a few metal-poor stars. Heavy r-process elements around the second peak compared to the Sun appear to show a universal sawtooth pattern, albeit with some internal variance, in the range $56<Z<75$ (Sneden et al. 2000; Cayrel 2001; Hill et al. 2002). But Otsuki, Mathews, \& Kajino (2003) stress that this does not imply a universality of abundances outside this range, and in fact this is not observed. Hill et al. (2002) find that the third peak and actinide elements $(Z \geq 90)$ are decoupled from elements in the second peak. There may be a substantial number of suitable elements (10-20) which could define a sufficiently large parameter space.

The very large scatter of [n-capture/Fe] in ultra metalpoor stars seen in Figure 12 of Wallerstein et al. (1997) means that n-capture element abundances in ultra-metalpoor stars are products of one or very few prior nucleosynthesis events that occurred in the very early, poorly mixed galactic halo, a theme that has been developed by many authors (e.g. Gilroy et al. 1988; Audouze \& Silk 1995; McWilliam et al. 1995; Shigeyama \& Tsujimoto 1998; Argast et al. 2000; Tsujimoto, Shigeyama, \& Yoshii 2000).

The simulation in Figure 1 is illustrative of the expected abundance variation in successive generations of lowmass stars in a closed box from gas enriched by successive generations of supernovae. The upper envelope is determined by enrichment from low-mass supernovae, the lower envelope from high-mass supernovae. From left to right, the black squares indicate the number of enrichment events, i.e. 1, 10, 100, 1000 events. Just ten enrichments from high-mass supernovae are sufficient to enrich a cloud to $[\mathrm{Fe} / \mathrm{H}]=-2$. Note the rapid convergence in this simple model above $[\mathrm{Fe} / \mathrm{H}]=-1$.

In the simulation, we consider only core collapse supernovae; subsequent enrichment by Type Ia supernovae causes the converging stream to dip down above $[\mathrm{Fe} / \mathrm{H}]=-1$. It is not clear at what $[\mathrm{Fe} / \mathrm{H}]$ the r-process elements become swamped by the ubiquitous Fe-group and s-process elements. Travaglio et al. (1999) suggest that the s-process does not become significant until $[\mathrm{Fe} / \mathrm{H}] \sim-1$ because of the need for pre-existing seed nuclei (Spite \& Spite 1978; Truran et al. 2001), although Pagel \& Tautvaisiene (1997) argue for some s-process production at $[\mathrm{Fe} / \mathrm{H}] \sim-2.5$. In any event, Figure 2 makes clear that, at sufficiently high resolution, both the Sun and Arcturus reveal a rich network of heavy elements, many of which arise from the r-process.

An important point to appreciate is that the substantially smaller scatter in $[\alpha / \mathrm{Fe}]$ (see Carretta et al. 2002), compared to heavy element scatter, presumably argues that the scatter is unlikely to arise from chemical differentiation, i.e. where metal yields from a given supernova are distributed in different amounts, compared to Fe, to the surrounding ISM. In other words, the mixing is sufficiently 

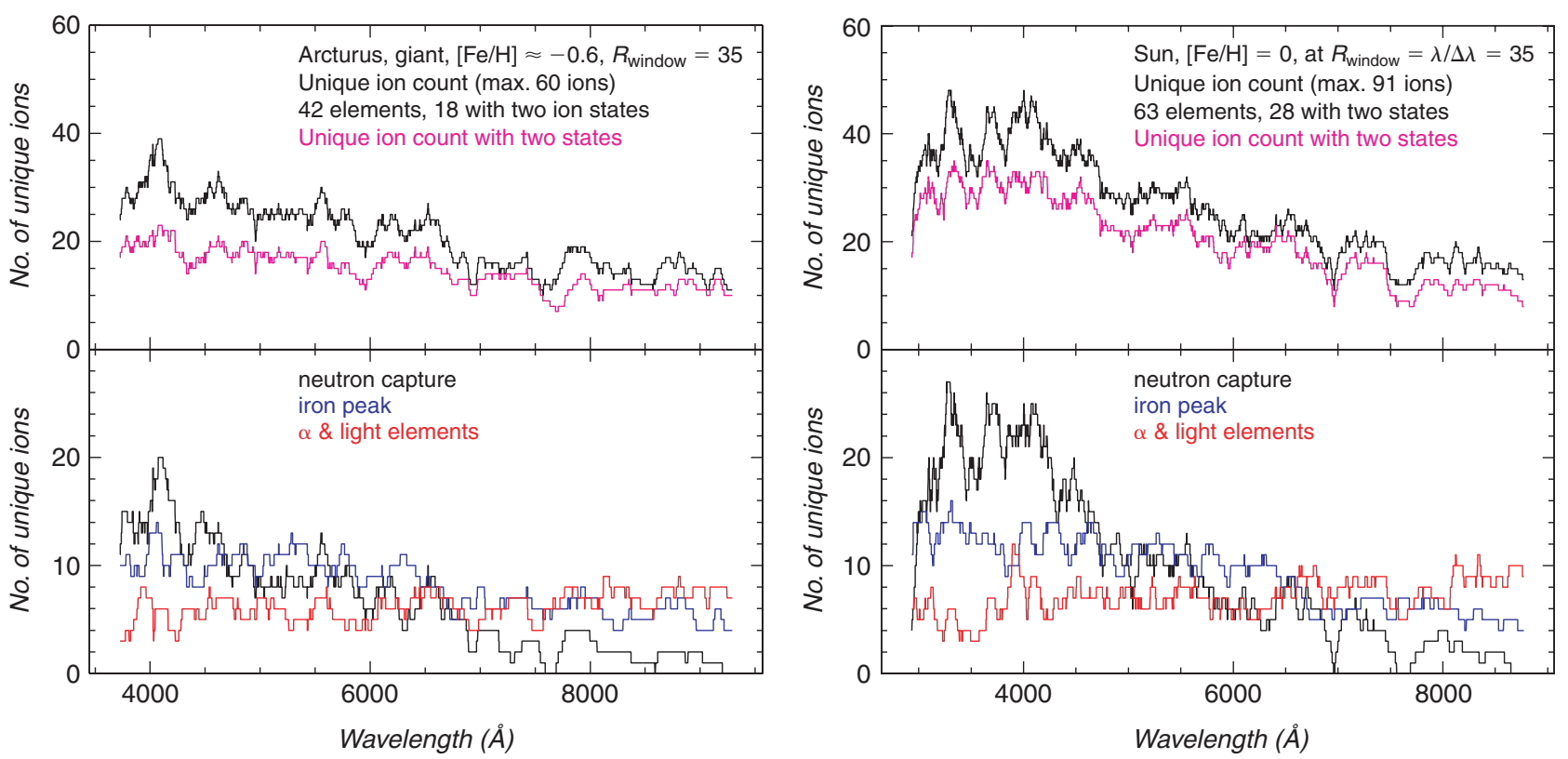

Figure 2 A demonstration of where most of the chemical information lies in a highly resolved spectrum of Arcturus (left) and the Sun (right). The black lines in the top plots give the total ion count in a spectral window $\Delta \lambda=\lambda / 35$. The magenta lines show what fraction of this count is made up of elements with two ionisation states. The lower plots separate these into element types. Note the rapid rise of heavy elements to the blue in both stars; note also that the solar spectral information extends to bluer wavelengths.

uniform such that any given cell receives the same proportion of $\mathrm{Eu} / \mathrm{Fe}$ from the supernova. This puts limits on the degree to which asymmetric supernovae (Maeda et al. 2002) must differentially enrich the surrounding gas.

Condition 5. Any practical experiment involving chemical signatures in millions of stars will require a wide-field, multi-fibre spectrograph operating at echelle resolutions. Since Rayleigh scattering renders optical fibres almost useless much below $4000 \AA$, it is important to establish that there exist contiguous spectral windows which contain abundance information on a large number of chemical elements. Since it will be necessary to maximise the number of object fibres at the detector, this information will need to exist within a single narrow band $(\sim \lambda / 35)$ echelle order. The optimal parameters for a 'galaxy genesis' machine are determined below.

Do there exist narrow band spectral windows, with chemical information on dozens of elements, which could be utilised by a fibre-fed echelle spectrograph? Until recently, this has not been possible to answer objectively. Figure 3 shows such a window that was revealed by this study. An optimal window needs information on more than one ionisation state for as many elements as possible in order to accurately determine the stellar surface gravity. Various ionic transitions in combination must also provide accurate information on the stars luminosity and surface temperature. Some regions of the spectrum are dominated by telluric features; in cool stars, molecular bands complicate the spectrum.

We concentrate our analysis on digitised atlases of the Sun $([\mathrm{Fe} / \mathrm{H}]=0.0)$ and of the luminous giant Arcturus
$([\mathrm{Fe} / \mathrm{H}]=-0.56)$ (McWilliam, Rich, \& Smecker-Hane 2003). Our solar line list derives from data supplied by P. Hall (NOAO) transcribed from the Moore, Minnaert, \& Houtgast (1966) solar spectrum (2935-8770 ̊). The atlas includes information on 25000 absorption lines covering 63 elements, for which 28 are detected in two ionisation states. The Arcturus line list comes from the digitised atlas (3570-7405 A) of Wallace, Hinkle, \& Livingston (1998). The line list includes 7700 atomic transition for 42 elements, for which 18 are detected in two ionisation states. Both lists are dominated by Fe I (45\%) and light $\alpha$ elements $(30 \%)$.

Figure 2 shows the number of unique ions detectable as a function of wavelength for the Sun and for Arcturus. At each wavelength, the accessible window is $\Delta \lambda$ where $\lambda / \Delta \lambda=35$, such that at $\lambda=3500 \AA$, the unique ion count is made over $\Delta \lambda=100 \AA$. The linear dependence of the window on wavelength is expected for spectral coverage in a single echelle order limited by the detector size. Note the rising count towards with decreasing wavelength down to $4000 \AA$, both in terms of the number of unique ions, and elements with two ionisation states.

In the lower panels of Figure 2, we demonstrate that this rise is largely due to a rising fraction of neutron capture elements; the rise in iron peak elements is not as dramatic. It is noteworthy that we obtain similar results for a solar metallicity main sequence star and a metal weak giant. Of particular importance to the KAOS project, note that there is an optimal window close to $4100 \AA$ accessible to optical fibres.

In Figure 4, we show the effect of resolving power on the fraction of lines which can be resolved as a function 

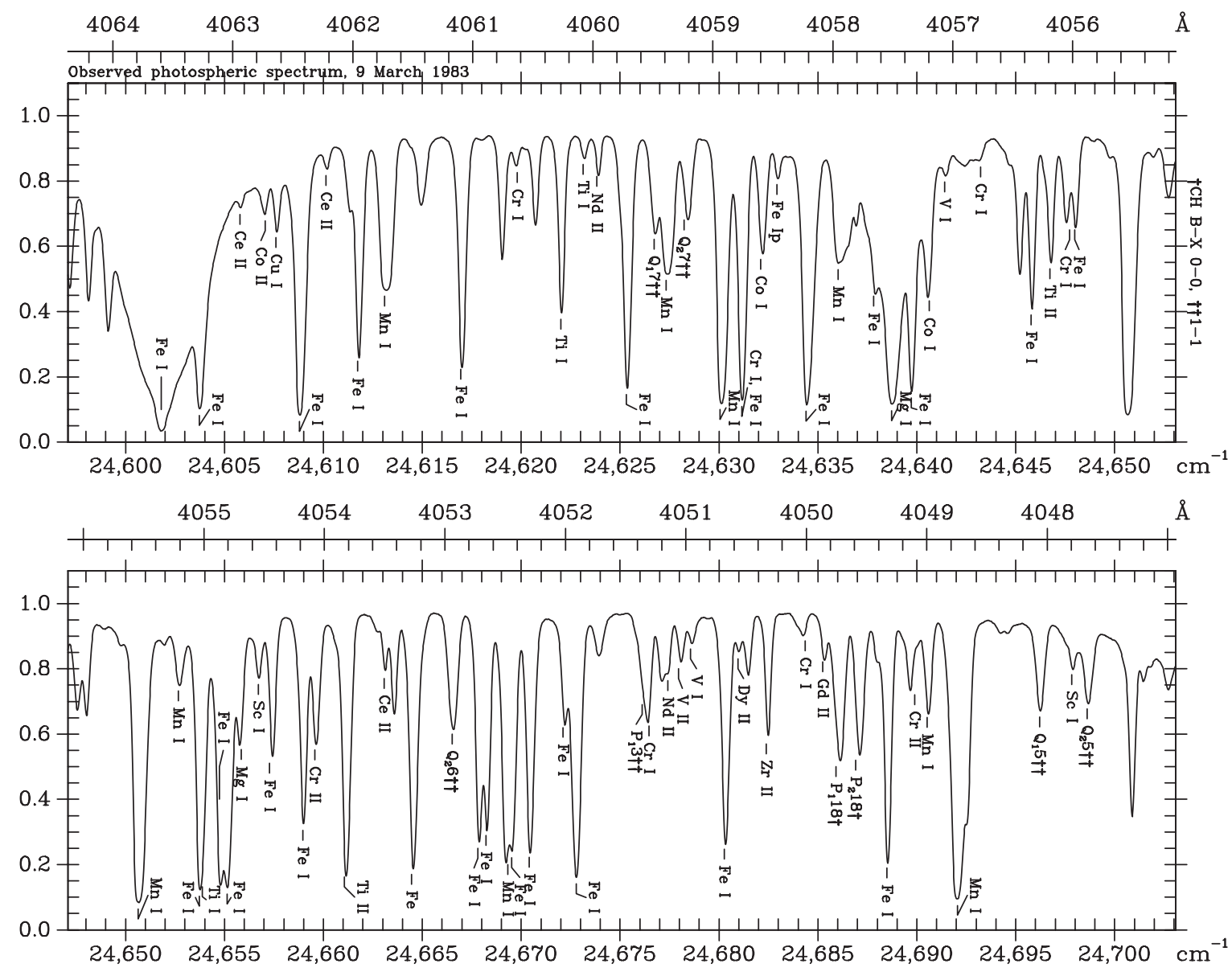

Figure $317 \AA$ A spectral window (4047-4064 ̊̊) of the Sun obtained with the Fourier Transform Spectrograph at the National Solar Observatory revealing detailed chemistry on $\mathrm{Fe}, \mathrm{Cr}, \mathrm{Ti}, \mathrm{V}, \mathrm{Co}, \mathrm{Mg}, \mathrm{Mn}, \mathrm{Nd}, \mathrm{Cu}, \mathrm{Ce}, \mathrm{Sc}, \mathrm{Gd}, \mathrm{Zr}$, Dy. The lower values are wavenumbers $\left(\mathrm{cm}^{-1}\right)$. The data are taken from http://www.nso.edu/.
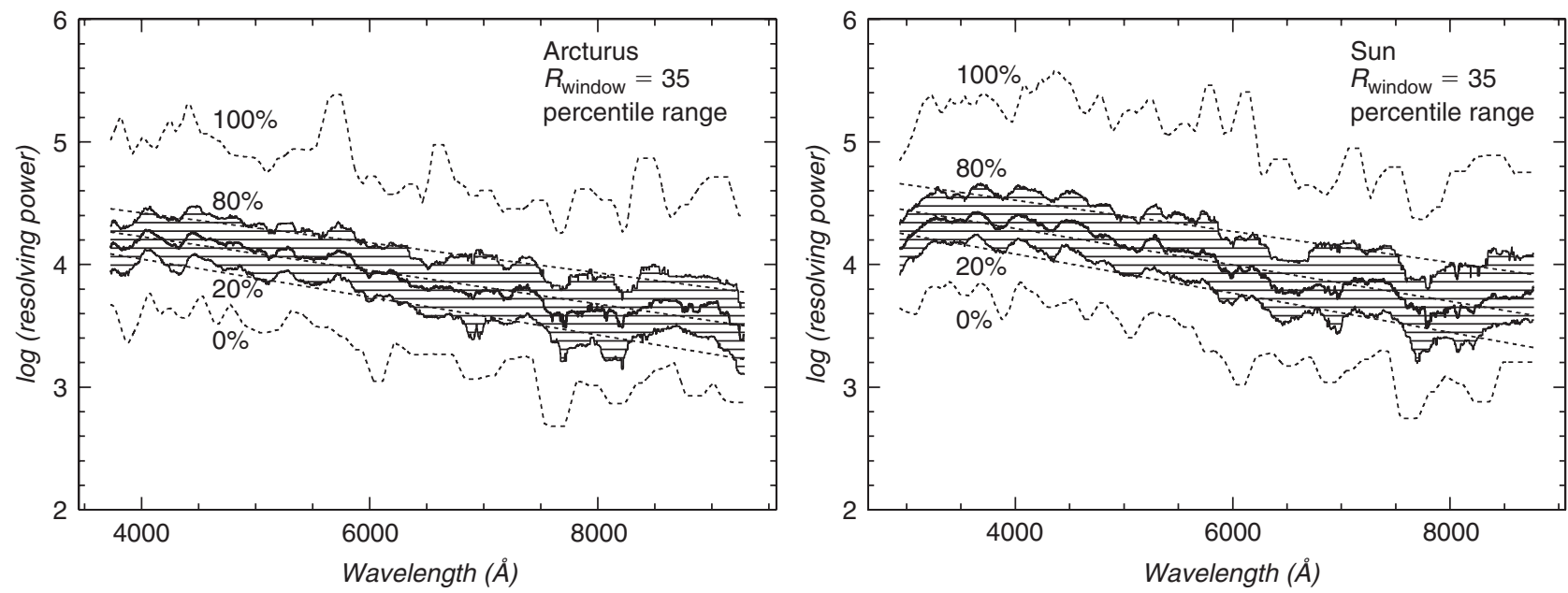

Figure 4 The effect of resolving power on the fraction of spectral lines which can be resolved as a function of wavelength. In order to resolve $80 \%$ of lines at $4000 \AA$, we need $R=40000$; this falls to $R=10000$ at $8000 \AA$. Once again, both stars reveal the same general trends; note also that the solar spectral information extends to bluer wavelengths.

of wavelength. The optimal window at $4100 \AA$ requires a spectral resolving power of $R=40000$ in order to resolve $80 \%$ of the lines. As the resolving power decreases, the loss of information on heavy elements is particularly dramatic.
We conclude that the KAOS Galaxy Genesis project should target $4100 \AA$ in a $100 \AA$ window at a resolving power of $R=40000$. The required sampling $0.05 \AA$ per pixel or 2000 pixels in the dispersion direction, well matched to state-of-the-art CCDs. 


\subsection{Candidates for Chemical Tagging}

Chemical tagging is not possible for all stars. In hot stars and young star clusters, our ability to measure abundances is reduced by the stellar rotation and lack of transitions for many ions in the optical. In very cool stars, very little light emerges in the optical or near-IR due to complex molecular and dust opacities. The ideal candidates are evolved FGK stars that are intrinsically bright, FGK subgiants and dwarfs. These populations probably account for $10 \%$ of all stars.

Giants can be observed at $R=40000$ over the full Gaiasphere, i.e. the $20-\mathrm{kpc}$ diameter sphere centred on the Sun which will be surveyed in great detail by the Gaia satellite. Dwarf stars will only be observable within $1-2 \mathrm{kpc}$. While this is only a small fraction of the available volume, the local volume may include a representative sample of all old disk stars, regardless of their point of origin.

For many stars, certain spectral regions are dominated by thermally broadened profiles, particularly for light elements. In general, heavier atoms show narrow profiles appropriate to their mass, although odd numbered atomic isotopes are susceptible to hyperfine splitting (due to the non-zero nuclear magnetic moment) which produces a broadened asymmetric line profile.

Giants have deep, low-density atmospheres that produce strong, low-ionisation absorption lines compared to higher gravity atmospheres. Even in the presence of significant line blending, with sufficient signal, it should be possible to derive abundance information by comparing the fine structure information with accurate stellar synthesis models. Detailed abundances of large numbers of F- and G-subgiants would be particularly useful, if it becomes possible to make such studies, because direct relative ages can be derived for these stars from their observed luminosities.

\section{Short-Term Goal - Size and Structure in a Multi-Dimensional $\mathcal{C}$-Space}

An intriguing prospect is that reconstructed star clusters can be placed into an evolutionary sequence, i.e. a family tree, based on their chemical signatures. Let us suppose that a star cluster has accurate chemical abundances determined for a large number $n$ of elements (including isotopes). This gives it a unique location in an $n$-dimensional space compared to $m$ other star clusters within that space. We write the chemical abundance space as $\mathcal{C}\left(\mathrm{Fe} / \mathrm{H}, \mathrm{X}_{1} / \mathrm{Fe}, \mathrm{X}_{2} / \mathrm{Fe}, \ldots\right)$ where $\mathrm{X}_{1}, \mathrm{X}_{2} \ldots$ are the independent chemical elements that define the space (i.e. elements whose abundances are not rigidly coupled to other elements).

Our simple picture assumes that a cloud forms with a unique chemical signature, or that shortly after the cloud collapses, one or two massive SNe II enrich the cloud with unique yields which add to the existing chemical signature. The low-mass population forms with this unique chemical signature. If the star-formation efficiency is high ( $\geq 30 \%)$, the star group stays bound although the remaining gas is blown away. If the star-formation efficiency is low, the star cluster disperses along with the gas. In a closed box model, the dispersed gas reforms a cloud at a later stage.

In the closed box model, each successive generation of supernovae produce stellar populations with progressive enrichments. These will lie along a trajectory in $\mathcal{C}$-space (see Fig. 8 of Karlsson \& Gustafsson 2001). The overall distribution of the trajectories will be affected by fundamental processes like the star-formation efficiency, the star-formation timescale, the mixing efficiency, the mixing timescale, and the satellite galaxy infall rate.

A critical test of chemical tagging is that stellar streams in the halo, identified from detailed phase space information, are highly localised in $\mathcal{C}$-space, or are confined to chemical tracks — this is a key short-term goal. There may already be evidence for accreted halo stars from their distinct chemical signatures (e.g. suppressed Mg) since those with estimated orbital parameters are found to have large apogalacticon distances (King 1997; Nissen \& Schuster 1997; Carney et al. 1997; Carretta et al. 2002). This may suggest that these stars originated in lower mass stellar systems with very different chemical histories from that of the Galaxy.

Our team (De Silva, Bland-Hawthorn, Freeman, Bessell, Asplund) is currently engaged in large surveys to establish the chemical homogeneity of open clusters as this forms a central tenet of 'chemical tagging'. We propose to extend this survey to large star-forming complexes which include several stellar associations within one or two super-associations. This is the sample volume we need to tackle to establish the existence of chemical tracks. Presently, it is difficult to identify a proper sample until phase-space information becomes available from Gaia, although Hipparcos has provided detailed candidacy for a few star forming complexes (de Bruijne, Hoogerwerf, \& de Zeeuw 2001).

As we approach solar levels of metallicity in $[\mathrm{Fe} / \mathrm{H}]$, the vast number of trajectories will tend to converge (see Fig. 21 of Wallerstein et al. 1997). By $[\mathrm{Fe} / \mathrm{H}] \sim-2.5$, AGB stars will have substantially raised the s-process element abundances; by $[\mathrm{Fe} / \mathrm{H}] \sim-1$, Type Ia supernovae will have raised the Fe-group abundances. Star clusters which appear to originate at the same location in this $\mathcal{C}$-space may reflect a common formation site, or the resolution limit we can expect to achieve in configuration space.

Even with a well established family tree based on chemical trajectories in the chemical $\mathcal{C}$-space, this information may not give a clear indication of the original location within the protocloud or Galactic component. This will come in the future from realistic baryon dissipation models. Forward evolution of any proposed model must be able to produce the observed chemical tree.

However, the $\mathcal{C}$-space will provide a vast amount of information on chemical evolution history. It should be possible to detect the evolution of the cluster mass function 
with cosmic time (Kroupa 2002), the epoch of a starburst phase and/or associated mass ejection of metals to the halo (Renzini 2002), and/or satellite infall (Noguchi 1998). The chemical tracks could conceivably be punctuated by discontinuities due to dramatic events like galactic infall or large-scale winds (Chiappini et al. 1999).

As we go back in time to the formation of the disk, we approach the chemical state laid down by population III stars. The rarity of stars below $[\mathrm{Fe} / \mathrm{H}] \sim-5$ suggests that the protocloud was initially enriched by the first generation of stars (Argast et al. 2000) or maybe that stars moving through the ISM today have a minimum threshold metallicity due to Bondi-Hoyle accretion. However, the apparent absence of any remnants of population III remains a puzzle: Its stars may have had a top-heavy initial mass function, or have dispersed into the intragroup medium of the Local Group. If one could unravel the abundances of heavy elements at the time of disk formation, this would greatly improve the precision of nucleo-cosmochronology. Important information is beginning to emerge from echelle observations of damped Ly $\alpha$ systems at high redshift (Pettini et al. 2003).

\subsection{The Size of $\mathcal{C}$-Space}

The size of $n$ is unlikely to exceed about 60 for the foreseeable future. Sneden et al. (2003) present exquisite data for the metal-poor star CS 22892-052, where abundance estimates are obtained for a total of 54 elements, with tight upper limits on three other elements. Hill et al. (2002) present exquisite data for the metal-poor star CS 31082001, where abundance estimates are obtained for a total of 44 elements, almost half the entire periodic table (see also Cayrel 2001; Sneden et al. 2001a,b). The $\alpha$ and r-process elements, and maybe a few canonical s-process elements at low $[\mathrm{Fe} / \mathrm{H}]$, provide information on the cloud abundances prior to star formation, although combinations of these are likely to be coupled (Heger \& Woosley 2001; Sneden et al. 2001a,b). There are more than two dozen r-process elements that have been clearly identified in stellar spectra (Wallerstein et al. 1997; Sneden et al. 2003).

The size of $m$ is likely to be exceedingly large for the thin disk where most of the baryons reside. For a rough estimate, we take the age of the disk to be $10 \mathrm{Gyr}$. If there is a unique SN II enrichment event every 100 years, we expect of order $10^{8}$ formation sites. Typically, a SN II event sweeps up a constant mass of $5 \times 10^{4}$ (Ryan, Norris, \& Beers 1996; Shigeyama \& Tsujimoto 1998). Simple chemical evolution models indicate that this must be of the right order to explain the metallicity dispersion at low [Fe/H] (Argast et al. 2000). Roughly speaking, there have been $10^{3}$ generations of clouds since the disk formed, with about $10^{5}$ clouds in each star-forming generation, such that cloud formation and dispersal cycles on a $10^{7} \mathrm{yr}$ timescale (Elmegreen et al. 2000).

Whereas the total number of star clusters over the lifetime of the thin disk is very large, the size of $m$ for the stellar halo (Harding et al. 2001), and maybe the thick disk (Kroupa 2002), is likely to be significantly smaller. Our primary interest is the oldest star clusters. Reconstructing star clusters within the thick disk is a particularly interesting prospect since the disk is likely to have formed within 1-1.5 Gyr of the main epoch of baryon dissipation (Prochaska et al. 2000).

\subsection{How Many Unique Chemical Signatures Comprise the Halo or the Thick Disk?}

There are different approaches to answering this question: (1) carry out a cold dark matter simulation (with hydro) and trace stellar evolution and feedback within each of the building blocks which make up a galaxy; (2) carry out a Press-Schechter simulation based on statistical aggregation with prescriptive physical laws; (3) undertake an order of magnitude calculation.

Our basic view is that modern CDM simulations in (1) are not able to carry out enough chemical information in order to do this properly. In its place, the numerical approach in (2) is currently under investigation by us: Our initial conclusion is that the expected number of signatures is very poorly defined due to uncertainties in supernova yields, and the sequence of events involved in star formation and recycling of gas. In place of detailed simulations, we provide an order of magnitude estimate.

There are at least three possible scenarios for the thick disk, all of which can be tested by chemical tagging. (1) The thick disk is thought to be a snap-frozen relic of the early disk, heated vertically by the infall of an intermediate mass satellite. This is a particularly interesting prospect since chemical tagging would provide clues on the formation of the first star clusters in the early disk. (2) Kroupa (2002) has suggested that the thick disk arose from a population of super-star clusters $\left(\sim 10^{6} \mathrm{M}_{\odot}\right)$ which became unbound. (3) Bekki \& Chiba (2000) have shown how the thick disk could arise from the infall of a number of Local Group satellites; see also Abadi et al. (2003). Their models could explain why thick-disk stars are more metal-rich than stars in dwarf galaxies or other likely building blocks, because the minor merger would have triggered star formation and self-enrichment.

For a thick disk made up of super-star clusters, we would need to detect roughly $10^{4}$ unique chemical signatures, requiring a survey of $10^{5}$ stars in order to detect a unique chemical signature at the $3 \sigma$ level. For a thick disk made up of the heated early disk, we would need to detect of order $10^{7}$ unique signatures, requiring a survey of $10^{8}$ stars. For a thick disk comprising roughly ten Local Group satellites, the number of unique chemical signatures will depend on the details of the star-formation history within these satellites prior to infall. But very roughly, we would need to detect $10^{6}$ unique signatures, requiring a survey of $10^{7}$ stars.

In the next section, in a more detailed statistical analysis, we show that a survey of $10^{6}$ stars may be sufficient to identify the chemical signatures of the early thick disk and the stellar halo. 
Table 1. The number of stars needed to detect progenitor sites (normalised to the number of sites)

The rows indicate the fraction of sites which are detected in 1, 2, 3, 5, 10, 30 stars. For example, if there are 1000 possible sites, and we want to detect 5 stars in $10 \%$ of these, we need to randomly sample about 2400 stars from the complete sample

\begin{tabular}{rrrrrrrrrr}
\hline & $10 \%$ & $20 \%$ & $30 \%$ & $40 \%$ & $50 \%$ & $60 \%$ & $70 \%$ & $80 \%$ & $90 \%$ \\
\hline 1 & 0.11 & 0.22 & 0.36 & 0.50 & 0.68 & 0.90 & 1.19 & 1.60 & 2.32 \\
2 & 0.53 & 0.81 & 1.08 & 1.36 & 1.67 & 1.99 & 2.41 & 2.97 & 3.87 \\
3 & 1.10 & 1.53 & 1.92 & 2.27 & 2.67 & 3.10 & 3.62 & 4.26 & 5.29 \\
5 & 2.43 & 3.09 & 3.61 & 4.13 & 4.65 & 5.21 & 5.88 & 6.73 & 8.08 \\
10 & 6.22 & 7.30 & 8.16 & 8.90 & 9.65 & 10.46 & 11.41 & 12.60 & 14.21 \\
30 & 23.20 & 25.30 & 26.92 & 28.33 & 29.64 & 31.05 & 32.69 & 34.56 & 37.21 \\
\hline
\end{tabular}

\subsection{Searching for Progenitor Formation Sites - How Many Stars Do We Need?}

Our simulations show that we need to identify roughly $10 \%$ of the original formation sites in order to properly sample cosmic time for a wide range of possible starformation histories (stochastic, exponential). In Table 1, we show the size of survey needed to detect a certain fraction of formation sites where the detection requires 1,2 , $3,5,10$, or 30 stars from each site.

Thick disk. If we adopt Kroupa (2002) model for the thick disk, we would need to survey 60000 thick-disk stars in order to establish $3 \sigma$ chemical signatures for $10 \%$ of the original formation sites. As we show below, this requires 500-1000 KAOS pointings in order to survey such a large number of thick-disk stars in a limited magnitude range.

Stellar halo. The baryon mass of the stellar halo is in the range $10^{8}-10^{9} \mathrm{M}_{\odot}$, depending on the details of how we distinguish between halo and bulge stars, and whether we include recently identified halo objects (e.g. Sgr dwarf). For illustration, we take the lower bound here, since we can scale trivially to the higher bound. Early universe simulations suggest that the first star clusters (e.g. protoglobulars, first dwarfs) must contain roughly a Jeans mass, or about $10^{5} \mathrm{M}_{\odot}$ in baryons (Abell, Bryan, \& Norman 2002). Since a single supernova can enrich a cloud of $10^{5} \mathrm{M}_{\odot}$, let us suppose that the Galactic halo arises from 1000 progenitor sites. Therefore, we would need to survey 500 halo stars to be sure of seeing the chemical signatures of more than one star from $10 \%$ of the original formation sites. With sufficiently accurate abundance information, a survey of 6000 halo stars would give a discrete chemical signatures $(3 \sigma)$ for $10 \%$ of the original sites.

If the original halo-formation sites saw several generations of star formation before infall, we should expect to see chemical tracks in $\mathcal{C}$-space. If we need 30 stars from a single formation site to establish a chemical track, we need to survey roughly 25000 halo stars in order to establish tracks for $10 \%$ of the progenitor sites. This amounts to 500-1000 KAOS pointings which can be observed simultaneously with the thick-disk pointings (see below).

If chemical tracks exist, it is fundamentally important to identify them because the starting point identifies a unique set of chemical signatures from a first-generation supernova. As mentioned above, there are a host of fundamental uncertainties about the enrichment details from supernovae, which severely limits our ability to perform realistic $\mathrm{CDM}+$ hydro simulations. But there is likely to be substantial progress in this arena (e.g. Gibson et al. 2003; Brook et al. 2004).

Note that the chemical tagging of the halo will be assisted by phase space information supplied by the KAOS survey and the Gaia space mission. It is presently unclear whether phase space information for the thick disk will provide supporting evidence for discrete groups identified by their unique chemical signatures.

In summary, a survey of 25000 halo stars and 60000 thick-disk stars may be sufficient to detect a useful number of progenitor formation sites; these observations will share 500-1000 KAOS pointings (see below).

\subsection{How Many Chemical Signatures Are We Ever Likely to Detect?}

The data to answer this question do not exist: stellar surveys to date target either too few stars or too few chemical elements. The answer depends in part on the strategy: we could attempt accurate measurements $(<0.05 \mathrm{dex})$ on a few decoupled lines, or try to distinguish, say, a high and a low state in many elements. An error of 0.02 dex is equivalent to $1 \mathrm{~m} \AA$ measurement accuracy in equivalent width.

Most physical processes are intrinsically noisy, so it would be surprising if there did not exist at least a small amount of decoupling in elements which appear to correlate within the measurement errors over a wide dynamic range in metallicity. The rapid statistical convergence with increasing $[\mathrm{Fe} / \mathrm{H}]$ seen in Figure 21 of Wallerstein et al. (1997) is assumed to arise from the increasing homogeneity of the ISM with cosmic time. But some intrinsic scatter could be sustained by gaseous infall (however see Pagel \& Tautvaisiene 1997).

In Figure 2, there are more than 30 unique elements observable in our optimal window. If we could distinguish a high and a low state for each of these, we could in principle distinguish a billion unique chemical signatures. Either strategy would require an accurate differential abundance 
Table 2. Distance limit in $\log ($ parsecs) for different stars as a function of apparent $V$ magnitude

Metal-poor giants (MPG), metal-rich giants (MRG), clump giants (CG), blue horizontal branch halo (BHB), and main sequence dwarfs. The second column is the absolute $V$ magnitude of the star. Brackets help to delineate the transition between 1-10-100 kpc. Note that the Solar Circle provides an extra $8 \mathrm{kpc}$ in radial extent such that surveys which reach the Galactic Centre also reach the outer disk

\begin{tabular}{lccccccccc}
\hline & $V$ & 13 & 14 & 15 & 16 & 17 & 18 & 19 & 20 \\
\hline MPG & -2.0 & $(4.0)$ & 4.2 & 4.4 & 4.6 & 4.8 & $(5.0)$ & 5.2 & 5.4 \\
& -1.5 & 3.9 & 4.1 & 4.3 & 4.5 & 4.7 & 4.9 & 5.1 & 5.3 \\
MRG & -1.0 & 3.8 & $(4.0)$ & 4.2 & 4.4 & 4.6 & 4.8 & $(5.0)$ & 5.2 \\
& -0.5 & 3.7 & 3.9 & 4.1 & 4.3 & 4.5 & 4.7 & 4.9 & 5.1 \\
& 0.0 & 3.6 & 3.8 & $(4.0)$ & 4.2 & 4.4 & 4.6 & 4.8 & $(5.0)$ \\
CG/BHB & 0.5 & 3.5 & 3.7 & 3.9 & 4.1 & 4.3 & 4.5 & 4.7 & 4.9 \\
& 1.0 & 3.4 & 3.6 & 3.8 & $(4.0)$ & 4.2 & 4.4 & 4.6 & 4.8 \\
& 1.5 & 3.3 & 3.5 & 3.7 & 3.9 & 4.1 & 4.3 & 4.5 & 4.7 \\
A & 2.0 & 3.2 & 3.4 & 3.6 & 3.8 & $(4.0)$ & 4.2 & 4.4 & 4.6 \\
& 2.5 & 3.1 & 3.3 & 3.5 & 3.7 & 3.9 & 4.1 & 4.3 & 4.5 \\
& 3.0 & $(3.0)$ & 3.2 & 3.4 & 3.6 & 3.8 & $(4.0)$ & 4.2 & 4.4 \\
F & 3.5 & 2.9 & 3.1 & 3.3 & 3.5 & 3.7 & 3.9 & 4.1 & 4.3 \\
& 4.0 & 2.8 & $(3.0)$ & 3.2 & 3.4 & 3.6 & 3.8 & $(4.0)$ & 4.2 \\
& 4.5 & 2.7 & 2.9 & 3.1 & 3.3 & 3.5 & 3.7 & 3.9 & 4.1 \\
G & 5.0 & 2.6 & 2.8 & $(3.0)$ & 3.2 & 3.4 & 3.6 & 3.8 & $(4.0)$ \\
& 5.5 & 2.5 & 2.7 & 2.9 & 3.1 & 3.3 & 3.5 & 3.7 & 3.9 \\
& 6.0 & 2.4 & 2.6 & 2.8 & $(3.0)$ & 3.2 & 3.4 & 3.6 & 3.8 \\
& 6.5 & 2.3 & 2.5 & 2.7 & 2.9 & 3.1 & 3.3 & 3.5 & 3.7 \\
K & 7.0 & 2.2 & 2.4 & 2.6 & 2.8 & $(3.0)$ & 3.2 & 3.4 & 3.6 \\
& 7.5 & 2.1 & 2.3 & 2.5 & 2.7 & 2.9 & 3.1 & 3.3 & 3.5 \\
\hline
\end{tabular}

analysis (Edvardsson et al. 1993; Prochaska et al. 2000; Paulson, Sneden, \& Cochran 2003).

But we stress that it may not be necessary to measure as many as 30 elements if some can be found which are highly decoupled and exhibit large relative dispersions from star to star. Burris et al. (2000) demonstrate one such element pair, i.e. $[\mathrm{Ba} / \mathrm{Fe}]$ versus $[\mathrm{Sr} / \mathrm{Fe}]$, confirmed by Carretta et al. (2002) who also suggest $[\mathrm{Mn} / \mathrm{Fe}]$ versus $[\mathrm{Cr} / \mathrm{Fe}]$ may have appreciable dispersion. It is part of our short-term goal to establish which axes in $\mathcal{C}$-space have the largest dispersions, and which axes are likely to be most decoupled.

\section{Long-Term Goal - Reconstructing Ancient Star Groups from Unique Chemical Signatures}

The abundance dispersion in $\alpha$ and heavy elements provides a route forward for tagging groups of stars to common sites of formation. With sufficiently detailed spectral line information, it is feasible that the "chemical tagging' will allow temporal sequencing of a large fraction of stars in a manner analogous to building a family tree through DNA sequencing.

Consider the (extraordinary) possibility that we could put many coeval star groups back together over the entire age of the Galaxy. This would provide an accurate age for the star groups either through the colour-magnitude diagram, or through association with those stars within each group that have $[\mathrm{n}$-capture/Fe] $\gg 0$ and can therefore be radioactively dated. This would provide key information on the chemical evolution history for each of the main components of the Galaxy.

There is no known age-metallicity relation that operates over a useful dynamic range in age and/or metallicity. (This effect is only seen in a small subset of hot metal-rich stars.) Such a relation would require the metals to be well mixed over large volumes of the ISM. For the foreseeable future, it seems that only a small fraction of stars can be dated directly $(\mathrm{FBH})$.

Ideally, we would like to tag a large sample of representative stars with a precise time and a precise site of formation. Can we identify the formation site? The kinematic signatures will identify which component of the Galaxy the reconstructed star group belongs, but not specifically where in the Galactic component (e.g. radius) the star group came into existence. For stars in the thin disk and bulge, the stellar kinematics will have been much affected by the bar and spiral waves; it will no longer be possible to estimate their birthplace from their kinematics. Our expectation is that the derived family tree will severely restrict the possible scenarios involved in the dissipation process. In this respect, a sufficiently detailed model may be able to locate each star group within the simulated time sequence.

Our ability to detect structure in $\mathcal{C}$-space depends on how precisely we can measure abundance differences between stars. It may be possible to construct a large database of differential abundances from echelle spectra, with a precision of $0.05 \mathrm{dex}$ or better; differential abundances are preferred here to reduce the effects of systematic error. 


\subsection{The Observing Programme}

We seek to target old stars in the thick disk and halo. In order to target a large enough volume of the thick disk and halo, we need reach to an apparent mag $V=17$ (equivalent to $I \sim 16.5$ ); see Table 2 . We propose to observe stars in a narrow magnitude range, $15<V<17$, in order to reduce the effects of scattered light in the instrument (Wyse \& Gilmore 1992, 1995).

If we adopt Gilmore's model (Gilmore, Reid, \& Hewitt 1985) of the Galaxy (see also Robin et al. 2003), within our magnitude range towards the Galactic poles there are

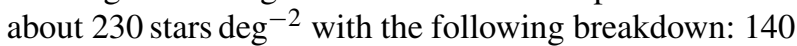
thin-disk main sequence (MS), 45 thick disk MS, 10 halo MS, 15 evolved (subgiant or red giant) thick disk, 15 evolved halo, 5 halo horizontal branch. Along a cardinal sight line of $\left(l=90^{\circ}, b=30^{\circ}\right)$, these numbers increase to 820 stars $\mathrm{deg}^{-2}$, with the following breakdown: 660 thindisk MS, 80 thick disk MS, 10 halo MS, 4 evolved thin disk, 43 evolved (subgiant or red giant) thick disk, 15 evolved halo, 5 halo horizontal branch. This amounts to 60 thick-disk stars towards the poles, and 120 stars at the lower latitude; we observe about 30 halo stars along either sight line.

A reasoned SNR calculation for a high-resolution KAOS design (see web calculator at http://www.noao.edu/ kaos) shows that we should be able to achieve SNR $\sim 100$ per pixel at $V=17 \mathrm{in} \mathrm{a} 4 \mathrm{hr}$ exposure $(R \sim 20000)$ rising to $\mathrm{SNR} \sim 300$ at $V=15$. Here we assume a $0.5^{\prime \prime}$ seeing observation of a $3000-\mathrm{K}$ blackbody source at $5000 \AA$ in full moon. A median SNR $\sim 150$ for the survey will be sufficient to identify unique chemical tracks and chemical signatures. This level of performance is encouraging for $R \sim 40000$ performance if this could be factored into the KAOS design.

For sanity, we have checked the predicted performance against a UVES commissioning observation (single slit, not fibre fed) which claims SNR 100 at $4000 \AA$ for $I \sim 15$ star at $R \sim 25000$ in one-hour exposure. This is a factor of two better than KAOS as expected since KAOS is fibre fed.

The actual spectroscopic resolution we need to achieve depends in part on the spectrophotometric stability, both in terms of an accurate zero level to each spectrum, and an accurately calibrated instrument response. If both zero level and instrument response can be calibrated accurately and consistently, it may be possible to conduct the KAOS survey at $R=20000$. However, if, say, the zero level can be maintained, but not the instrument response correction, it is safer to work at $R=40000$, and fit individual lines in order to measure equivalent widths. But the advantage of removing the instrument response accurately is that it assists in determining the true continuum level by matching to atmospheric models. If more than one order is observed, instrument response correction is also important for joining orders together.

In summary, for the benchmark KAOS one degree field, we detect 60000 thick-disk stars in 500-1000 KAOS fields, and 25000 halo stars in about 1000 KAOS fields. Ideally, we would opt for $R \sim 40000$ but it may be possible to work at $R \sim 20000$ with sufficient instrument stability.

\section{Related Surveys and the Way Ahead for Near-Field Cosmology}

Detailed high-resolution abundance studies of large samples of galactic stars will be crucial to the success of the Galaxy Genesis project. The KAOS survey is one of three massive stellar surveys.

The first is the RAVE survey (see http://www.iap.de/ RAVE) which will obtain radial velocities and abundances for 50 million stars, i.e. all stars down to $V=16$, over the period 2005-2010 (Steinmetz 2002). This requires a new generation 'Echidna' spectrograph mounted on a Schmidt telescope (1.2-m aperture), with 2250 fibres working at a spectroscopic resolution of $R \sim 8000$.

By far the most ambitious proposal is the Gaia satellite mission (see http://www.rssd.esa.int/GAIA) over the period 2012-2018 which is expected to launch during the time frame of the KAOS survey (Perryman et al. 2001). This will provide photometric, spectroscopic and phase space information for a billion stars in the Local Group. The Gaia mission is expected to obtain $R \sim 10000$ resolution spectroscopy for up to 100 million stars or more.

It is important to note several points about the Gaia and RAVE surveys: these are based on snapshot surveys with a $1-\mathrm{m}$ aperture telescope, targeting the $8700 \AA$ region at low resolving power. Their choice of $R=8000$ (RAVE) and $R=10000$ (Gaia) are supported by Figure 4. As we have seen, this region carries limited abundance information. Both missions are primarily optimised for kinematic studies. The KAOS echelle survey will be the first million star survey optimised for chemical abundance work.

The KAOS survey requires a highly multiplexed, highresolution spectrograph which will be expensive and technically challenging, but we believe this must be tackled if we are to ever unravel the formation of the Galaxy. Could some of the residual inhomogeneities from prehistory have escaped the dissipative process at an early stage? We may not know the answer to this question with absolute certainty for many years. But it is an intriguing thought that one day we may be able to identify the Solar Family, i.e. the hundreds or even thousands of stars throughout the Gaiasphere that were born within the same cloud as the Sun.

\section{References}

Abell, T., Bryan, G. L., \& Norman, M. L. 2002, Science, 295, 93 Abadi, M. G., Navarro, J. F., Steinmetz, M., \& Eke, V. R. 2003, ApJ, 597, 21

Argast, D., Samland, M., Gerhard, O. E., \& Thielemann, F.-K. 2000 A\&A, 356, 873

Audouze, J., \& Silk, J. 1995, ApJL, 451, 49

Bekki, K., \& Chiba, M. 2000, ApJL, 534, 89

Brook, C. B., Kawata, D., Gibson, B. K., \& Flynn, C. 2004, MNRAS, 349,52 
Burbidge, E. M., Burbidge, G. R., Fowler, W. A., \& Hoyle, F. 1957, Reviews of Modern Physics, 29, 547

Burris, D. L., Pilachowski, C. A., Armandroff, T. E., Sneden, C., Cowan, J. J., \& Roe, H. 2000, ApJ, 544, 302

Carney, B. W., Wright, J. S., Sneden, C., Laird, J. B., Aguilar, L. A., Latham, D. W. 1997, AJ, 114, 363

Carpenter, J. M. 2000, AJ, 120, 3139

Carretta, E., Gratton, R., Cohen, J. G., Beers, T. C., \& Christlieb, N. 2002, AJ, 124, 481

Cayrel, R. et al. 2001, Nature, 409, 691

Chiappini, C., Matteucci, F., Beers, T. C., Nomoto, K. 1999, ApJ, 515,226

Clarke, C. J., Bonnell, I. A., \& Hillenbrand, L. A. 2000, in Protostars and Planets IV, eds. V. Mannings, A. P. Boss, \& S. S. Russell, 151 (Tucson: Univ of Arizona Press)

Clayton, D. 1988, MNRAS, 234, 1

Conti, P. S., Wallerstein, G., \& Wing, R. F. 1965, ApJ, 142, 999

Dalcanton, J. J., \& Bernstein, R. A. 2002, AJ, 124, 1328

de Bruijne, J. H. J., Hoogerwerf, R., \& de Zeeuw, P. T. 2001, A\&A, 367,111

Edvardsson, B., Andersen, J., Gustafsson, B., Lambert, D. L., Nissen, P. E., \& Tomkin, J. 1993, A\&A, 275, 101

Elmegreen, B. G., Efremov, Y., Pudritz, R. E., \& Zinnecker, H. 2000, in Protostars and Planets IV, eds. V. Mannings, A. P. Boss, \& S. S. Russell, 179 (Tucson: Univ of Arizona Press)

Feltzing, S., Bensby, T., \& Lundstrom, I. 2003, A\&A, 397, L1

Freeman, K. C., \& Bland-Hawthorn, J. 2002, ARA\&A, 40, 487

Fuhrmann, K. 1998, A\&A, 338, 161

Gibson, B. K., Fenner, Y., Renda, A., Kawata, D., \& Lee, H.-c. 2003, PASA, 20, 401

Gilmore, G., Wyse, R. F. G., \& Kuijken, K. 1989, ARA\&A, 27, 555

Gilmore, G., Wyse, R. F. G., \& Jones, B. 1995, AJ, 109, 1095

Gilmore, G., \& Reid, I. N. 1983, MNRAS, 202, 1025

Gilmore, G., Reid, I. N., \& Hewett, P. 1985, MNRAS, 213, 257

Gilroy, K., Sneden, C., Pilachowski, C. A., \& Cowan, J. J. 1988, ApJ, 327, 298

Goriely, S., \& Arnould, M. 1996, A\&A, 312, 327

Harding, P., Morrison, H. L., Olszewski, E. W., Arabadjis, J., Mateo, M., Dohm-Palmer, R. C., Freeman, K. C., \& Norris, J. E. 2001, AJ, 122, 1397

Heger, A., \& Woosley, S. E. 2001, ApJ, 567, 532

Hill, V. et al. 2002, A\&A, 387, 560

Karlsson, T., \& Gustafsson, B. 2001, A\&A, 379, 461

King, J. R. 1997, AJ, 113, 2302

Kroupa, P. 2002, MNRAS, 330, 707

Luck, R. E., \& Bond, H. E. 1985, ApJ, 292, 559

Maeda, K., Nakamura, T., Nomoto, K., Mazzali, P. A., Patat, F., \& Hachisu, I. 2002, ApJ, 565, 405

Manuel, O. 2000, in Origins of Elements in the Solar System (Kluwer: New York)

McKee, C. F., \& Tan, J. C. 2002, Nature, 416, 59

McWilliam, A., Preston, G. W., Sneden, C., \& Searle, L. 1995, AJ, 109,2736

McWilliam, A., Rich, M. R., \& Smecker-Hane, T. A. 2003, ApJL, 592,21

Moore, C. E., Minnaert, M. G. J., \& Houtgast, J. 1966, The Solar Spectrum $2935 \AA$ to $8770 \AA$ (NBS Monogr. 61) (Washington: NBS)

Nissen, P. E., \& Schuster, W. J. 1997, A\&A, 326, 751
Noguchi, M. 1998, Nature, 392, 253

Norris, J. E., Ryan, S. G., \& Beers, T. C. 1996, ApJS, 107, 391

Otsuki, K., Mathews, G. J., \& Kajino, T. 2003, NewA, 8, 7670

Pagel, B. E. J. 1965, Royal Obs Bull, 104, 127

Pagel, B. E. J, \& Tautvaisiene, G. 1997, MNRAS, 288, 108

Paulson, D. B., Sneden, C., \& Cochran, W. D. 2003, AJ, 125, 3185

Perryman, M. A. C., de Boer, K. S., Gilmore, G., Høg, E., Lattanzi, M. G., Lindegren, L., Luri, X., Mignard, F., Pace, O., \& de Zeeuw, P. T. 2001, A\&A, 369, 399

Pettini, M., Madau, P., Bolte, M., Prochaska, J. X., Ellison, S. L., \& Fan, X. 2003, ApJ, 594, 695

Prochaska, J. X., Naumov, S. O., Carney, B. W., McWilliam, A., \& Wolfe, A. M. 2000, AJ, 120, 2513

Quillen, A. C. 2002, submitted to AJ (astro-ph/0202253)

Rauscher, T., Heger, A., Hoffman, R. D., \& Woosley, S. E. 2002, ApJ, 576, 323

Renzini, A. 2002, in Chemical Enrichment of Intracluster and Intergalactic Medium, ASP. Conf. Ser., 253, eds. F. Matteucci, \& R. Fusco-Femiano, 331 (San Francisco: ASP)

Robin, A. C., Reylé, C., Derriére, S., \& Picaud, S. 2003, A\&A, 409, 523

Ryan, S. G., Norris, J. E., \& Beers, T. C. 1996, ApJ, 471, 254

Schwarzkopf, U., \& Dettmar, R.-J. 2000, A\&A, 361

Shigeyama, T, \& Tsujimoto, T. 1998, ApJL, 507, 135

Sneden, C., Cowan, J. J., Ivans, I. I., Fuller, G. M., Burles, S., Beers, T. C., \& Lawler, J. E. 2000, ApJL, 533, 139

Sneden, C., Cowan, J. J., Beers, T. C., Truran, J. W., Lawler, J. E., \& Fuller, G. 2001a, in Astrophysical Ages and Time Scales, ASP Conf. Ser., 245, eds. T. von Hippel, C. Simpson, \& N. Manset, (San Francisco: ASP), 235

Sneden, C., Lawler, J. E., \& Cowan, J. J. 2001b, Physica Scripta, 100,15

Sneden, C. et al. 2003, ApJ, 591, 936

Spite, M., \& Spite, F. 1978, A\&A, 67, 23

Stahler, S. W., Palla, F., \& Ho, P. T. P. 2000, in Protostars and Planets IV, eds. V. Mannings, A. P. Boss, \& S. S. Russell (Tucson: Univ of Arizona Press), 327

Steinmetz, M. 2002, in GAIA Spectroscopy, Science \& Technology, ASP Conf. Ser., 298, ed. U. Munari (San Francisco: ASP), 381

Travaglio, C., Galli, D., Gallino, R., Busso, M., Ferrini, F., \& Straniero, O. 1999, ApJ, 521, 691

Tsujimoto, T., Shigeyama, T., \& Yoshii, Y. 2000, ApJL, 531, 33

Tsujimoto, T., \& Shigeyama, T. 1998, ApJL, 508, 151

Truran, J. W. 1981, A\&A, 97, 391

Truran, J. W., Burles, S., Cowan, J., \& Sneden, C. 2001, in Astrophysical Ages and Time Scales, ASP Conf. Ser., 245, eds. T. von Hippel, C. Simpson, \& N. Manset (San Francisco: ASP), 226

Wallace, L., Hinkle, K., \& Livingston, W. 1998, An Atlas of the Spectrum of the Solar Photosphere from 13500 to $28000 \mathrm{~cm}^{-1}$ (3570 to $7405 \AA$ ) (Tucson: NOAO)

Wallerstein, G., Greenstein, J. L., Parker, R., Helfer, H. L., \& Aller, L. H. 1963, ApJ, 137, 280

Wallerstein, G. et al. 1997, Rev. Mod. Phys., 69, 995

Wyse, R. 2000, in The Galactic Halo, from Globular Clusters to Field Stars, eds. A. Noels et al. (Liege: Institut d'Astrophysique et de Geophysique), 305

Wyse, R., \& Gilmore, G. 1992, AJ, 104, 144

Wyse, R., \& Gilmore, G. 1995, AJ, 110, 2771 